Authors have nothing to disclose with regard to commercial support.

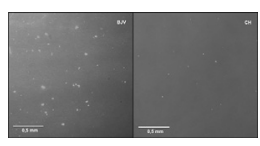

\section{ARE PLASMA \\ PROTEINS KEY \\ PLAYERS IN THE \\ PATHOGENESIS OF \\ INFECTIVE \\ ENDOCARDITIS?}

\section{Reply to the Editor:}

Infective endocarditis (IE) after the implantation of a bovine jugular vein (BJV) graft in the right ventricular outflow tract has recently been described to be more frequent than for other conduits. ${ }^{1}$ Yet, the exact mechanisms that drive this phenomenon are under discussion. Jalal and colleagues ${ }^{2}$ were the first to study bacterial adhesion to different tissues in vitro; their clinical bacterial isolates displayed increased adherence to BJV valves, as compared with bovine and porcine pericardium, especially in traumatized valve leaflets. As discussed in their accompanying letter, we have presented results somewhat incongruous with their findings by claiming that the surface composition of the conduits, bacterial surface proteins, and shear forces per se are not the most important determinants of bacterial adherence in vitro.

In this study, we primarily investigated whether (1) morphologic tissue differences, (2) bacterial species specificity, (3) exposure to extracellular substrates, and (4) stasis versus shear stress determine bacterial adhesiveness to the graft tissues investigated. However, as an advantage, in vitro models allow investigations in a comprehensive way, building up (patho)physiological complexity, progressively including more in vivo determinants.
We agree with the comment of Jalal and colleagues ${ }^{2}$ that clinical bacterial isolates may be more virulent than our reference strains and that the experimental setup influenced testing of bacterial adhesion in static or flow conditions. Nevertheless, the same Staphylococcus aureus, Staphylococcus epidermidis, and Streptococcus sanguis "lab" strains were tested in previous in vitro studies and manifested the full pathogenic spectrum of $S$ aureus, ie, activation of endothelial proinflammatory and procoagulant pathways, crucial in the onset of IE. ${ }^{4}$ Bacterial recruitment in the host is a more complex event. ${ }^{5}$ In addition to the adhesive factors presently studied, it is also determined by plasma proteins, platelets, and inflammatory cells to allow formation of a vegetation, indeed consisting mainly of fibrin and bacteria. In agreement with Jalal and colleagues, ${ }^{2}$ we admit that the exact in vivo conditions are difficult to replicate in an experimental setup and that IE models are required that will enable a more complex environment needed to better understand the mechanisms behind the clinical observations.

Therefore, to progressively evaluate factors present in the "in vivo status," as a first step, we have investigated the role of plasma proteins in bacterial adhesion to tissues in our flow models. First results show that fibrinogen adsorption was greater for the BJV tissue compared with the cryopreserved homograft tissue (Figure 1). We speculate that surface-bound fibrinogen might facilitate subsequent bacterial adhesion, as fibrin(ogen), together with fibronectin are known key players for the adhesion of IE-associated pathogens to traumatized endothelium or medical devices.

In agreement with Jalal and colleagues, ${ }^{2}$ we appreciate that further efforts will have to be undertaken to investigate the impact of plasma proteins, blood cells, and rheological factors on bacterial adhesiveness in conditions of laminar and turbulent flow. Such an approach may in turn foster the option of adequate IE-preventive therapy

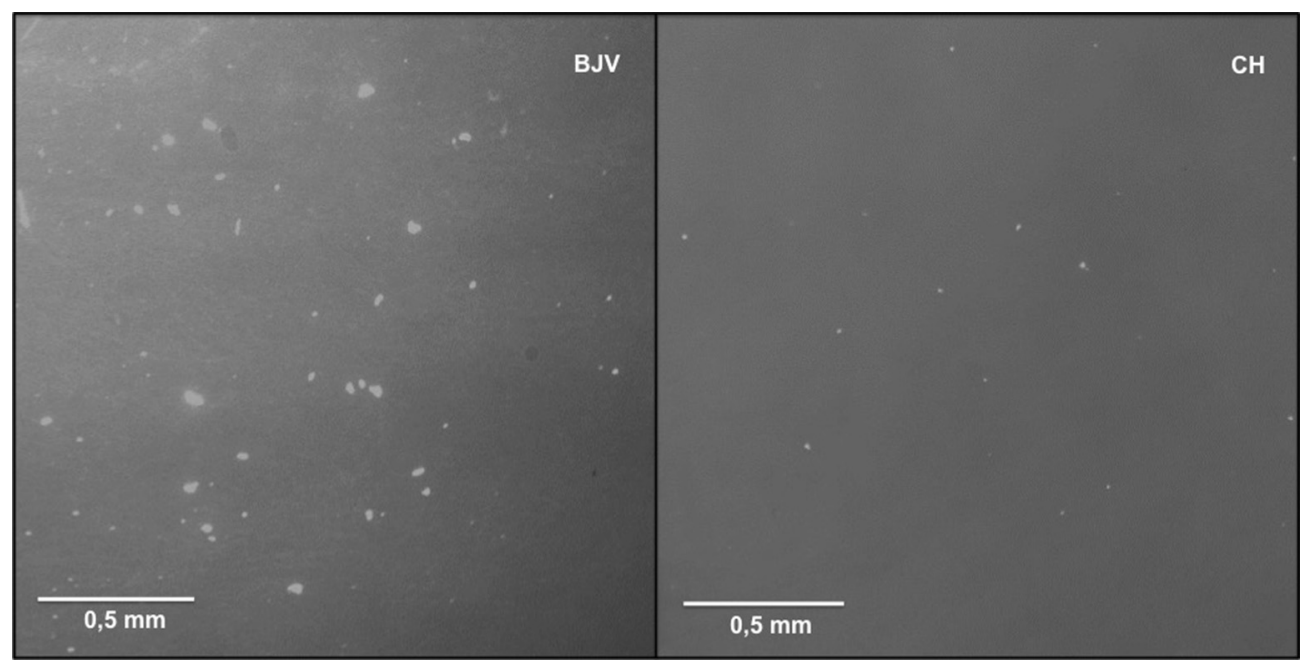

FIGURE 1. Deposition of fluorescently labeled fibrinogen (white foci) on $\mathrm{BJV}$ and $\mathrm{CH}$. 
and limit IE-associated risks to help increase patients' quality of life.

Tiago Rafael Veloso, PhD
Bartosz Ditkowski, PhD
Petra Mela, PhD
Marc F. Hoylaerts, PhD
Ruth Heying, MD, PhD
a
${ }^{b}$ Department of Tissue Engineering \& Textile Implants
AME-Helmholtz Institute for Biomedical Engineering
Aachen, Germany
Department of Cardiovascular Sciences
${ }^{c}$ Center for Molecular and Vascular Biology
Department of Cardiovascular Sciences
KU Leuven
Leuven, Belgium

\section{References}

1. Malekzadeh-Milani S, Ladouceur M, Patel M, Boughenou FM, Iserin L, Bonnet $\mathrm{D}$, et al. Incidence and predictors of $\operatorname{Melody}(\mathrm{R})$ valve endocarditis: a prospective study. Arch Cardiovasc Dis. 2015;108:97-106.

2. Jalal Z, Galmiche L, Lebeaux D, Villemain O, Brugada G, Patel M, et al. Selective propensity of bovine jugular vein material to bacterial adhesions: an in-vitro study. Int J Cardiol. 2015;198:201-5.

3. Veloso TR, Claes J, Van Kerckhoven S, Ditkowski B, Hurtado-Aguilar LG, Jockenhoevel S, et al. Bacterial adherence to graft tissues in static and flow conditions. J Thorac Cardiovasc Surg. 2018;155:325-32.e324.

4. Heying R, Wolf C, Beekhuizen H, Moelleken ML, Jockenhoevel S, Hoylaerts MF, et al. Fibrin- and collagen-based matrices attenuate inflammatory and procoagulant responses in human endothelial cell cultures exposed to Staphylococcus aureus. Tissue Eng Part A. 2011;18:147-56.

5. Liesenborghs L, Verhamme P, Vanassche T. Staphylococcus aureus, master manipulator of the human hemostatic system. J Thromb Haemost. 2018;16: 441-54.

https://doi.org/10.1016/j.jtcvs.2018.04.053

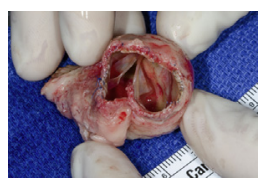

ELUCIDATING THE MECHANISMS OF INFECTIVE ENDOCARDITIS IN BOVINE JUGULAR VEIN CONDUITS: ARE WE ANY CLOSER?

\section{Reply to the Editor:}

Multiple reports have raised concerns regarding the high rates of late endocarditis in bovine jugular vein (BJV) grafts used for right ventricular outflow tract reconstruction as conduits $^{1,2}$ (Figure 1) or as percutaneously placed stentmounted valves. ${ }^{3,4}$ The pathophysiologic mechanisms for these findings remain unclear.

In a recent study in the Journal, Veloso and colleagues ${ }^{5}$ found that in vitro adherence of bacterial strains to patches

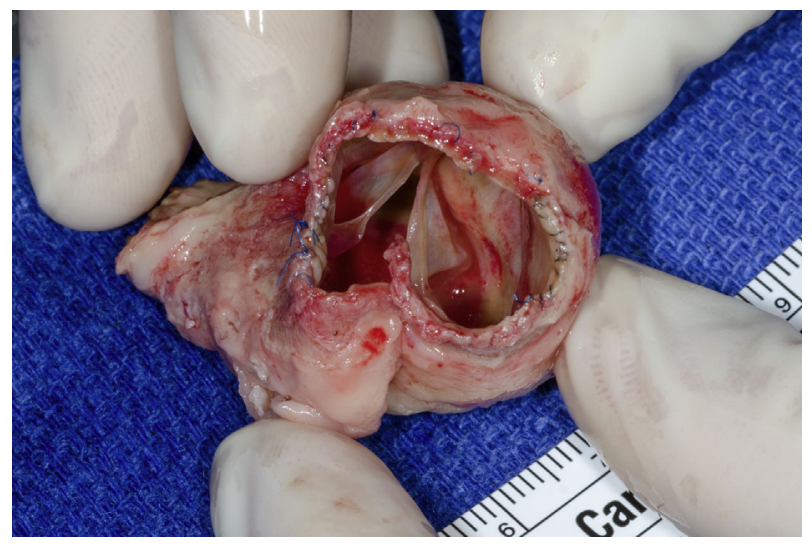

FIGURE 1. Necrotic bovine jugular conduit explanted 1 year after implantation.

of bovine pericardium, BJV, and cryopreserved homograft was similar between materials under both static and simulated flow conditions. These findings were different from those reported by Jalal and colleagues, ${ }^{6}$ who found that static bacterial adherence was greater in BJV tissue (including stent-mounted valves) than in porcine pericardium, and that adherence increased when the leaflets of the valves were traumatized. In a Letter to the Editor in this issue of the Journal, Jalal and colleagues ${ }^{7}$ suggest that the differences between both studies are likely explained by the use of native strains in the study by Veloso and colleagues ${ }^{5}$ compared with pathogenic strains isolated from patients in the study from Jalal and colleagues. ${ }^{6}$

Both studies are important steps toward the goal of elucidating the mechanisms that cause an increased clinical risk of endocarditis in patients receiving BJV grafts. However, they also illustrate the difficulty of simulating in the laboratory what happens to patients in the clinical setting, especially in the long term. The incidence of endocarditis for patients with BJV seems to be a late-occurring event, with the risk apparently increasing after 7 years from implantation. ${ }^{1}$ Endocarditis is likely a consequence of a complex interplay of multiple factors that include, among other things, material characteristics, bacterial strains, circulating coagulation factors and proteins, patient susceptibility, shear stress, turbulent flow, thrombosis, tissue injury, and time. Simulating this complex interplay in vivo is obviously quite challenging. Bacterial adherence is just one factor. In fact, some studies have shown that in vitro adherence may not correlate as well with the ability of a bacterial strain to cause experimental endocarditis in an in vivo model. ${ }^{8}$

It is clear that more sophisticated in vitro constructs may provide a better insight into the role that different bacterial strains and materials play in the development of endocarditis in real patients. The use of fluorescent-labeled bacteria and flow chambers to simulate shear stress like the ones used by Veloso and colleagues ${ }^{5}$ is a step on the right 